\title{
Las familias entre fines educativos y económicos. El caso de Chile
}

\section{Families between educational and economic ends. The case of Chile}

Propuesta

\author{
José Miguel Olave \\ Universidad de Chile - Chile \\ jose.olave@uchile.cl \\ Claudia Vélez de La Calle \\ Universidad San Buenaventura - Colombia \\ cvelez02@yahoo.es
}

Recibido: 7 de noviembre de 2016 / Aceptado: 15 de diciembre de 2016

\section{Resumen}

Este artículo problematiza la privatización de la educación en el contexto latinoamericano de mediados del siglo $X X$, cuando los Estados promueven la idea del capital humano por medio del crecimiento de sus sistemas educativos y la productividad como una razón de Estado. Las familias del continente creen en la promesa que les asegura que por medio del acceso a la educación se encontrarán la ansiada movilidad social. Confirmamos que para el inicio del siglo XXl el cumplimiento de esta promesa recae únicamente en los hombros de las familias quedando estas entregadas al mercado.

Sostenemos nuestra argumentación bajo el análisis de las diversas normativas que afectan al sistema educativo chileno en el periodo que va de 1860 a 2008 En ellas observamos particularmente la normativa en Evaluación Educativa, que va marcando este tránsito como un mecanismo de gobierno de la población, regulando los flujos sociales, traspasando, por medio de herramientas del mercado, la responsabilidad a las familias para escoger entre fines formativos y resultados académicos.

Finalmente concluimos con algunos alcances producto de este fenómeno que obliga a las familias a una doble renuncia: la renuncia económica que impone la inversión en educación por sobre otros proyectos alternativos; y la renuncia que implica dejar sus propias formas significativas de relacionarse al interior de las familias, por la adopción de una cultura del éxito escolar. Esta elección reafirma la matriz de gobierno colonial instalada sobre la familia y la vez la convierte en una unidad productiva eficiente sobre una idea de mérito neoliberal transmitido por medio de las evaluaciones.

Descriptores: Sociedad, cultura, familia, Chile, educación, evaluación educativa, decolonial.

\section{Abstract}

This article is part of the process of problematization on the privatization of education in Latin America with a special focus on the Chilean case. It analyzes elements of the Latin American context in the middle of the 20th century when States promote the idea of human capital through the growth of their education systems and productivity as a State reason. The families of the continent grow the promise that through access to education the long-awaited social mobility will be achieved. We confirm that at the beginning of the 2 I st century this promise falls only on the shoulders of families being left to the market.

We hold our argument under the analysis of various regulations affecting the Chilean educational

Forma sugerida de citar: Olave, José \& Vélez, Claudia (2017). Las familias entre fines educativos y económicos. El caso de Chile. Alteridad, 12(1), pp. 43-54. 
system in the period between I 860 and 2008. In these we observe in particular the regulations in Educational Evaluation, which is marking this transit as a mechanism of government of the population, regulating social flows, transferring through market tools, responsibility to families to choose between training purposes and academic results.

Finally we conclude with some scope resulting from this phenomenon that forces the families to a double resignation. The economic waiver imposed by investment in education over other alternative projects. And the renunciation of leaving their own significant ways of relating to the interior of families, by adopting a culture of school success. This election reaffirms the matrix of colonial government installed on the family and at the same time makes it an efficient productive unit on an idea of neoliberal merit transmitted through assessment.

Keywords: Society, culture, education, family, Chile, assessment educational, decolonial.

alimentada por un legítimo deseo de crear una buena vida para sus seres queridos.

En el caso de Latinoamérica tal promesa de superación por medio de la educación se vuelve un relato aceptado pero vaciado de realidad, dada la evidente brecha de desigualdad y concentración económica que cubre al mundo globalizado y que, en particular, se muestra con cierta indolencia en la realidad latinoamericana. Según cifras de CEPAL (2011, 2013) el nivel de desigualdad de América Latina y El Caribe es bastante alarmante. Se plantea que el ingreso de la quinta parte más rica es 19,7 veces mayor que el ingreso del $20 \%$ más pobre; en este contexto, una de cada tres personas es pobre, con variaciones que van desde países donde los pobres superan el 50\% de la población como Bolivia, Paraguay, Honduras o Guatemala, a otros en los que el porcentaje de pobres es inferior al 20\% como Costa Rica, Uruguay y Chile.

Adelantando el desarrollo del artículo, afirmamos que esta aspiración de superación vía acceso a la educación, solo ha provocado una ampliación del mercado educativo y ha dejado sobre los hombros de las familias los costos de tan preciada promesa. Las familias fueron invitadas a integrarse al deseo de cobertura en educación primaria y secundaria, y en la actualidad también lo hacen como demandantes de educación terciaria; demanda bien recibida por el sistema privado educativo, dado que de esta manera se consolida un creciente mercado a partir de la reinstitucionalización de las Universidades (Bernasconi y 
Fernández, 2012) para cubrir la demanda. Este problema tiene una raíz profunda en la neoliberalización de los sistemas educativos, que aprovechan este envión de la demanda, quedando el grueso de la inversión en manos de las familias, tal cual lo indica el reporte Panorama Educación Indicadores de la OCDE 2014.

En este informe se señala que la inversión en educación es altamente rentable (nótese el lenguaje). Para un hombre su inversión en educación tendría un retorno económico de US 100 000. No sería lo mismo para una mujer ya que su retorno sería de US 60000 , lo cual ya constituye una brecha de desigualdad. Además se afirma que:

El nivel educativo influye en las oportunidades de trabajo y los ingresos, que a su vez afectan al nivel de salud. La educación puede también mejorar los conocimientos sobre cómo vivir una vida más saludable, lo que lleva a mejores elecciones del uso del tiempo y de los bienes que afectan la salud (OCDE, 2014, p. 35).

Lo que no dice el informe es que en países como los de Latinoamérica, los niveles de precarización de los trabajos han aumentado y que los servicios, como educación, vivienda y salud, no son subsidiados por el Estado, es decir, son las personas, individualmente consideradas, las que tienen que pagarlos de sus bolsillos.

Este reporte sitúa a Chile como el país con mayor porcentaje de gasto privado en todos los niveles educativos dentro de los países miembros de esta organización económica: un $40 \%$ del gasto en educación proviene de las familias, cifra que se dispara al $76 \%$ en la educación terciaria (OCDE, 2014b). Asimismo, esta política de desresponsabilización de los Estados cae sobre los sectores más sensibles de la vida social y económica. Esta es parte de una política que tiene como consigna, menos Estado y más mercado, afectando los ámbitos básicos de la vida de la población: salud, vivienda, transporte público, pensiones de vejez, entre otros. En Chile, en el caso de la educación, se genera una mercado educativo multisegmentado, ya que no todos pueden pagar los colegios privados, es decir, quien tiene más puede pagar más, quien tiene poco, accede a poco y quien tiene menos que poco, se va a la educación pública

Se observa en consecuencia un panorama desregulado en el mercado educativo o, más bien, una suerte de aplicación maximizada de la "mano invisible del mercado", que deja a Chile no solo como el país con el mayor gasto privado en educación, sino además como uno de los países con mayor desigualdad social y económica (OCDE, 2013; CEPAL, 2011). Chile, según el último informe de desigualdad emitido por la OCDE (2014a) es el país con mayor índice de desigualdad de los países miembros de esta organización económica internacional con un 0,53 en el coeficiente GINI, donde el valor 0 indica mayor igualdad y 1 es el valor de mayor desigualdad 1 .

Todo lo anterior, no solo genera inequidad en relación a la posibilidad de pago de la educación, sino además genera una suerte de ethos social de la competencia, reafirmando la idea de que solo con virtudes individuales se puede asegurar el anhelado progreso social y económico, confirmando el sentido del mérito neoliberal.

La comprensión de la dimensión de nuestras preocupaciones la trabajaremos en un primer apartado donde situaremos la emergencia de esta promesa de la educación como vía de mejoramiento que marca la vida de las familias en Latinoamérica. En segundo lugar, buscaremos en la relación entre educación y evaluación un camino metodológico para explorar las normativas en el ámbito educativo y, en particular, sus reglamentos de evaluación, que son los que orientan la conducción de las familias hasta el momento actual, en el que se encuentran abandonadas a la suerte del mercado. Finalmente ofreceremos reflexiones sobre las implicancias de este proceso privatizador.

Ver más en http://data.oecd.org/inequality/income-inequality.htm 


\section{Fundamentación: educación latinoamericana, una promesa heredada}

Para comprender mejor la emergencia de este relato sociocultural que hemos llamado "La promesa latinoamericana", se debe mirar a la generación de los sesenta quienes crecen con la América Latina del proyecto desarrollista tardío que impulsa Estados Unidos (1961) en la región, el cual propuso un programa de ayuda económica y social denominado "Alianza para el Progreso", como respuesta a la revolución cubana de corte izquierdista; este consideraba mejorar las condiciones de vida de la población y se basaba en un riguroso sistema de planificación social:

Ya en la década de los sesenta y extendiéndose a lo largo de la siguiente, se crea y se difunde en el contexto de Estados Unidos y Europa, el pensamiento de la teoría del capital humano que, sin duda, marca las prioridades de la época. Para decirlo muy sencillamente, esta teoría intenta vincular la inversión en educación y su rendimiento, definiendo a la educación como un servicio con valor económico (Carlino, 1999, p.51).

Dado este contexto político, económico y social, en la Conferencia de Punta del Este (1961) los delegados de todos los países miembros de la Organización de Estados Americanos (OEA) acuerdan su adhesión a dicho plan (Alianza para el Progreso); excluyéndose Cuba, quien se niega a firmar el pacto vislumbrando el condicionamiento geopolítico que enmarca la situación de Latinoamérica hasta la época actual.

La política económica comienza a copar todos los espacios de la vida cotidiana y se pone en marcha una política de regulación nacional promovida desde un Estado que aplica estrategias de conducción cimentadas en principios regulatorios modernos (De Sousa, 2011, 2013), marcados por la dualidad totalidad/individualidad que se aplican en materia económica. Es la promoción del Estado asistencialista o benefactor (totalidad), quien se asume como motor de inversión creando estrategias de pleno empleo, impulsando la industria nacional, los servicios y la administración estatal, mientras que los ciudadanos (individualidad) que participan como trabajadores en el mercado también ahora lo harán como consumidores. Se produce un régimen de verdad reconocido por los ciudadanos, que aceptan la promesa moderna que su integración al mundo del trabajo les permitirá superar la desigualdad económica, y de que por medio de la educación y las políticas sociales del Estado, podrán integrarse, gracias a sus esfuerzos familiares, a esta pequeña totalidad construida.

La escuela asumirá el desafío de superar la desigualdad y la exclusión, consolidándose como la mayor institución regulatoria del Estado. Esta situación se expresa en la reforma de los años sesenta, bajo el Decreto 27.952 del año 1965, reforma que será frenada por las dictaduras latinoamericanas. Es tiempo de recesión económica, violencia política y dictaduras en el Cono Sur, incluyendo por cierto la dictadura en Chile de Pinochet (1973-1990). Es tiempo del viraje hacia las políticas neoliberales, entonces, la promesa Latinoamericana tendrá un condimento distinto, aunque manteniendo su sello original, la fe en la educación. Lo distintivo será que ahora los Estados latinoamericanos van a asumir un rol de promotores de la oferta educativa, tal como se define en la Constitución Política del Estado de Chile de 1980, ${ }^{2}$ donde el Estado debe garantizar el derecho a la enseñanza, es decir, hacer florecer el mercado educativo. Cambia el papel que asumen los Estados: desde un Estado providencial (Santos, 2003) que asume la dirección del proyecto desarrollista, a un Estado que media entre el mercado y los ciudadanos.

Para fines del siglo XX las orientaciones emanadas por organizaciones de distinta naturaleza tendrán un poderoso ascendiente en el diseño de políticas educativas tanto en Chile como en el conjunto de los países de Latinoamérica.

2 Ver más en http://muw.leychile.cl/Navegar?idNorma $=242302$ 
Un ejemplo de esta política regional es el informe Transformación productiva con equidad (CEPALUNESCO, 1990) y el documento Educación y conocimiento: eje de la transformación productiva con equidad (CEPAL-UNESCO, 1992), que expresan la relación entre sistema educativo y sistema productivo, dejando ver un marcado tono economicista para entender el fenómeno educativo como un proceso que debe maximizar sus resultados al menor costo posible.

Luego de la noche dictatorial de los ochenta (en Argentina, Uruguay, Chile y Brasil), llega un tiempo de grandes acuerdos en los países de la región para consolidar sus sistemas democráticos a través de la inversión en sus sistemas educativos. Es un período de plena influencia neoliberal en la política educativa que deja ver la relación insoslayable entre la economía política de financiamiento educativo y las vinculaciones entre educación y rendición de cuentas. En el caso de Chile, la dictadura de Pinochet termina con un proceso de gran movilización social que provoca que en 1988 se realice un plebiscito que da paso al retorno a la democracia. En marzo de 1990, el Dictador no entrega el poder sin antes promologar la última de las leyes llamadas de "amarre", la Ley Orgánica Constitucional de Educación (LOCE, 1990) promulgada un día antes del traspaso de mando.

Resumiendo este apartado, la promesa latinoamericana tiene su emergencia en las décadas de los cincuenta y sesenta del siglo pasado junto al proyecto desarrollista asociado a la idea del "capital humano", propia de la estrategia hegemónica de los Estados Unidos y Europa, para consolidar su control geopolítico en Latinoamérica. Más adelante, se instala la confianza en que el mercado y su productividad se basan en una racionalidad económica que los Estados recogerán a pie firme, desarrollando una política económica aplicada a la vida de la población, impactando con especial precisión en el campo educativo, asociándolo a la veleidosa idea de la promoción social para quienes participen de esta promesa.

\section{Metodología: la evaluación en una promesa privatizada}

Tal apuesta, en su inicio, es promovida por los Estados latinoamericanos a partir de estrategias de gobierno de la población, asumiendo un papel de corresponsabilidad junto a las familias. Su objeto de gobierno será la productividad de la población. Sin duda, podemos hablar aquí con propiedad de una conducción biopolítica (Foucault 2004, 2005) que a partir de estrategias de control sobre la población se intensifica, visibilizando la productividad como una razón de Estado. El fin de siglo estuvo marcado por el abandono por parte del Estado de su rol promotor de esta estrategia, abriendo espacio a otras estrategias más invisibles para imponer la relación entre el mercado, los sistemas educativos y las familias. En este sentido, se remarcan los costos económicos que esta tozuda oferta implica, aspectos que han sido suficientemente estudiados desde los ámbitos de la reproducción, la segregación y la desigualdad; abordajes que han silenciado otros costos que las familias deben pagar.

Con el fin de analizar el fenómeno de privatización que afecta al sistema educativo chileno, el que incide directamente en las familias, la metodología que utilizaremos revisa las principales normativas desde el inicio de la República de Chile en 1860 hasta el año 2008 (ver Tabla Anexo 1) con objeto de ilustrar cómo estas normativas afectan al sistema educativo chileno. Este análisis documental de primera fuente es combinado con la revisión de literatura de autoras que han avanzado en este camino. En específico, se investiga sobre las disposiciones en evaluación que van agenciando las decisiones de las familias por el acceso a un determinado nivel educativo, gobernando sus vidas por medio del acceso o no, a distintos niveles educativos, ya que entendemos, al igual que la autora Teresa Flórez (2012), que:

Hasta hoy el poder de la evaluación, refleja su capacidad para hacer que todo el sistema educativo se mueva en una dirección específica. Hay 
mucho que perder y que ganar como para ignorarla, por lo que más allá de cualquier principio o ideal pedagógico y, por lo tanto, de cualquier idea de sociedad que se quiera desarrollar, es la evaluación de alto riesgo la que define las reglas para la práctica y la que decide, finalmente, los destinos de nuestra sociedad (p. 84).

En Chile, al igual que en otros países de Latinoamérica, la educación previa a la Independencia fue mayormente una preocupación de las organizaciones religiosas. Con el paso de la Independencia a las repúblicas emergentes, el control de la educación por parte de las nuevas naciones se constituye en un efecto legitimador. El naciente Estado promovió la creación de establecimientos educativos que imprimieran los valores de la nueva Nación, como el Instituto Nacional $(1813)^{3}$ creado por José Miguel Carrera, luego de la primera Junta de Gobierno celebrada en 1810. La idea era crear un sistema educativo que fomentara los idearios de la naciente Nación chilena y alojara a la elite criolla de la época. Este establecimiento es el primer liceo chileno público de varones y, hasta hoy, es quien lidera los rankings de efectividad para entrar a la universidad y, por lo tanto, acceder a él también constituye el principal objetivo para las familias, con vistas a la promoción social. Es uno de los liceos a los que en Chile se les llama coloquialmente "emblemáticos" por su larga tradición pública y republicana.

El control de la educación por parte del Estado se llevó a cabo a través de estas instituciones, quedando las organizaciones educativas religiosas supeditadas a rendir examen de validación ante comisiones evaluadoras representantes del sistema estatal, tarea que le fue encomendada a la naciente Universidad de Chile (1942) ${ }^{4}$. La fundación de la Universidad de Chile en 1842 es, sin duda alguna, uno de los hitos más significativos de la naciente República. Representa en sí uno de los más claros actos de autodeterminación,

\footnotetext{
Ver más en http://institutonacional.cl/el-instituto/

4 Ver más en http://uww.uchile.cl/portal/presentacion/historia/ resena-historica/4727/una-mirada-a-la-historia
}

al situar en el saber y la enseñanza pública el eje articulador del país que comienza a construirse. Existía consenso entre los intelectuales de la época de que un Estado - aun no estando separado de la Iglesia como en ese entonces- debía velar por el progreso y la promoción de valores universales. Desde un comienzo la Universidad de Chile se define a sí misma como garante de la cultura clásica, humanista y secular.

A partir de la Ley Orgánica para la Instrucción Pública de 1860, se comenzó a regular el sistema educativo en dos segmentos, educación primaria y educación secundaria, con este instrumento normativo. Se ordena el flujo educativo que tendría la población chilena, distribuyéndola funcionalmente. A partir de los exámenes, un tipo de estudiante terminaría sus estudios en la primaria para incorporarse a las tareas productivas; los que logran pasar el examen, principalmente los hijos de las elites, podrían seguir estudios para luego optar por una de las profesiones liberales para el servicio del Estado naciente. Se observa que para el nivel primario su énfasis era la educación moral y la alfabetización de la población. El segundo nivel se componía de las escuelas preparatorias, escuelas secundarias e instituciones de la educación superior -dependientes de la recién creada Universidad de Chile- dirigidas a la formación de profesionales y a la elite burocrática. El tono que marca este periodo es la especial preocupación por la educación secundaria, por el contrario, se observa un cierto desprecio por la educación primaria, entendida como la educación de las clases populares. Se imprime en ella el tono civilizatorio ilustrado que identifica esta época en Latinoamérica:

La única autoridad que tienen los docentes es sobre los estudiantes, quienes son descritos frecuentemente como si se tratara de salvajes provenientes de familias incivilizadas, en el contexto de una época en que la disciplina, el silencio y el orden eran considerados parte fundamental de la educación (Flórez, 2012, p. 11).

En síntesis se observa una regulación de orientación centralizada y verticalista, que por 
medio de sus instituciones regula y controla los flujos de la sociedad. Además, este periodo inicial se caracteriza por un marcado afán civilizatorio y de baja consideración pedagógica.

En un primer momento, en la primera mitad del siglo XX, en Chile se percibe la influencia de las ideas educativas representantes de la "escuela nueva", centrada en las experiencias educativas de los niños y niñas, lo que implica mirar la relación entre profesor y estudiantes de otra manera; intención que se observa en la Ley de Obligatoriedad de la Educación Primaria (Ley $3.654,1920$ ), además de la reforma educativa de 1928 y 1929, principalmente promovida por los docentes de la época. En este periodo los reglamentos en evaluación se caracterizan por mantener una suerte de bajo control académico para la educación primaria (Decreto 3060, 1929; Circular No 77, 1934), no así para la educación segundaria (Decreto 6087, 1927; Decreto 2545, 1929; Decreto 2944, 1944; Decreto 1469, 1949 y Decreto 842, $19595)$. En estas normativas se observa un avance al sugerir que los exámenes no solo sean al final de cada periodo escolar para evitar que el juicio evaluativo sea reservado a un solo examen, entrando en vigencia la idea de un promedio de calificaciones. Para regular el acceso a la educación terciaria, se estableció en 1929 un solo examen administrado por la Universidad de Chile, el llamado examen de "Bachillerato", como un requisito de ingreso a todas las universidades. El Estado por medio de los exámenes busca asegurar procesos de socialización, remarcando la responsabilidad individual en el proceso educativo:

Debido a las prácticas de evaluación más objetivas, los resultados se conectaron cada vez más a título personal, las actitudes de los individuos y mérito, y los alumnos eran vistos cada vez más como responsables de su propio éxito o fracaso (Gysling, 2015, p. 6).

5 Para mayor profundidad, revisar el detalle de la normativa en evaluación en el trabajo realizado por Gysling (2015), donde se agrupan normativas y se las ordenadas bajo una estricta mirada crítica.
Un segundo momento en el siglo XX es el ingreso del discurso del capital humano, recogiendo la demanda por mayor cobertura y eficiencia. Se lleva a cabo la reforma educativa (1967) que va a administrar estos intereses agregando dos años de escolaridad obligatoria (de seis se pasa a ocho años de obligatoriedad) y nuevas materias escolares, reforzando un currículo escolar en el área de ciencias naturales y matemáticas. Asimismo, se introducen las técnicas de evaluación por selección múltiple y de esta manera se implementará en Chile una prueba única para seleccionar el ingreso a las universidades. La llamada Prueba de Aptitud Académica (PAA) fue entregada para su aplicación a la Universidad de Chile, la cual evaluaba las aptitudes verbales y matemáticas de los estudiantes siguiendo el modelo internacional SAT (por sus siglas en inglés, Test de Aptitudes Escolares) muy difundido en Estados Unidos e Inglaterra, que se utiliza para predecir resultados en la vida académica futura (Stobart, 2010). Este modelo supone que las aptitudes o capacidades se pueden desarrollar en un ambiente educativo donde el docente resulta un habilitador de estas capacidades, por lo tanto, se aleja del modelo innatista promovido por los test de inteligencia que suponen medir el coeficiente intelectual fijo. Se incorporó la evaluación criterial ${ }^{6}$ focalizada en materias escolares excluyéndose aspectos de conducta y de personalidad.

En materia de evaluación la normativa tendrá algunas innovaciones asociadas a la instalación de la reforma educativa de 1967 y que se expresan en el Decreto 11.207, 1967; Decreto 6.859, 1968; Decreto 7056, 1967 y el Decreto 192, 19727). Entre las principales modificaciones, se encuentra la elaboración de una prueba local, la cual será diseñada por grupos de maestros de distintas escuelas. La promoción estaba ligada solo a

6 La evaluación criterial corresponde a la pedagogía de dominio o mastery learning relacionada con la pedagogía por objetivos y el conductismo. Esta surge vinculada a la difusión de la enseñanza programada y el auge de los objetivos conductuales.

7 Ver mayor detalle en Gysling (2015). 
aspectos académicos siendo excluidos elementos de comportamiento moral.

Estas reformas de índole democratizador son interrumpidas por la irrupción de la dictadura de Pinochet (1973-1990) que se caracterizó por una gran represión a los movimientos y organizaciones sociales populares y de las clases medias. Se favorece el control de los contenidos y se le quita poder a los docentes, organizados antes en Departamentos, para generar sus pruebas locales y estas son reemplazadas por las pruebas globales que ahora son de responsabilidad individual de cada docente (Decreto supremo 164, 1974; 440, $1975 ; 2.038,1978 ; 2.088,19798)$. Específicamente el Decreto 2038, 1978, que aprueba el Reglamento de evaluación y promoción escolar para estudiantes de Educación General Básica (primaria) y Educación Media (segundaria), en su artículo número 1 de disposiciones generales, letra $\mathrm{C}$, establece:

Al término del año escolar, se aplicará una Prueba Global escrita a los estudiantes de $5^{\circ} \mathrm{a}$ $8^{\circ}$ año de Educación General Básica, en cada una de las asignaturas del respectivo Plan de Estudios (...). La prueba Global será elaborada por el respectivo profesor, conforme a los criterios establecidos en los Departamentos de asignaturas, correspondientes (Decreto Ley, 2038, www.Leychile.cl).

De acuerdo a esta normativa, se pierde la posibilidad de generar un juicio compartido en evaluación por parte de distintos maestros, por el contrario, se instala un juicio evaluativo individual, práctica evaluativa que responsabilizaría en mayor medida a estudiantes y profesores de sus éxitos y fracasos. Asimismo en este periodo, se debe mencionar que a la PAA creada en la etapa anterior (1975), se le agregan otras pruebas de conocimientos específicos, volviendo a la postura tradicional del dominio académico por sobre las aptitudes, lo cual ejerce mayor presión en el rendimiento individual para acceder a la universidad.

8 Ver http://www.leychile.cl/Navegar?idNorma $=251386$
En un tercer momento, a fines del siglo XX, se intensificará la política de apertura al mercado de abierta influencia neoliberal (Ruiz, 2010) y la toma de posición de un Estado Evaluador (Elliot, 2002), promotor de las evaluaciones nacionales estandarizadas, y que partir de ellas genera un complejo de incentivos económico, pudiendo comparar a las escuelas por medio de los resultados de este tipo de pruebas. El tiempo del "Estado Evaluador" abre el paso a una administración geopolítica de los resultados académicos, donde Chile y los demás Estados de Latinoamérica participan como condición de entrada a determinados mercados. En esta lógica, como mecanismo adicional, en Chile se fomenta el emprendimiento de escuelas particulares impulsadas por el financiamiento a la demanda o sistema de "voucher", nombre que se le da en Chile al subsidio a la demanda. Este sistema consiste en que a cada estudiante se le asigna un valor, que el Estado pagará a los establecimientos públicos y particulares subvencionados. Este sistema también se ocupa para movilizar el mercado de la salud o de la construcción, subsidiando a las familias que desean comprar su primera vivienda. Los "voucher" serán el mecanismo que moverá la competencia entre escuelas tratando de capturar la matrícula. Los estudiantes se convierten, así, en un valor que se agrega al negocio de la educación. La llamada prueba nacional SIMCE -sistema de medición nacional basado en estándares- será el índice que los padres podrán seguir para invertir en educación. Asimismo las escuelas competirán por capturar a los estudiantes y hacer cobro de los "voucher".

Finalmente, en una última normativa, la Ley Orgánica Constitucional de Enseñanza (LOCE, 1990) se contempla que los resultados de las pruebas nacionales se puedan publicar, impulsando un perverso sistema de competencia entre escuelas.

En un cuarto momento, al inicio del siglo XXI, la intensidad de las pruebas estandarizadas aumenta. En materia de evaluación Chile se integra prontamente a este sistema de control geopo- 
lítico de los resultados, participando en las pruebas Pisa, Timss y en el sistema de evaluaciones regionales promovido por OREALC/UNESCO. Pero, sin lugar a dudas, la característica principal del período es la ampliación en número y niveles de las pruebas nacionales. Este sistema resulta ser de multipropósito, de allí la evidente tensión entre los fines pedagógicos y su marcada funcionalidad como mecanismo para la rendición de cuentas. La ley de Garantía de Calidad (Ley 20.529, 2011) le concede al Estado la capacidad de intervenir e incluso cerrar las escuelas, dado un sistema de clasificación de escuelas basado principalmente en los resultados obtenidos en la prueba SIMCE.

Paralelamente, se aprueba una nueva normativa, la Subvención Escolar Preferencial ${ }^{9}$ (Ley, $\mathrm{N}^{\circ} 20.248,2008$ ), que asigna un nuevo subsidio a cambio de resultados académicos. La Ley de Subvención Especial Preferencia (SEP) se establece como una medida de equidad con el fin de entregar más recursos por medio del sistema, una vez más, del subsidio a la demanda ("voucher"). La mayor asignación se entrega a los estudiantes de sectores con mayor vulnerabilidad llamados estudiantes "prioritarios" una sutileza para categorizar la pobreza. En 2011 se integran cambios a esta ley, subiendo en un $21 \%$ el bono de asignación económica por estudiantes, a cambio de que las escuelas diseñen un plan de acción de mejora de resultados académicos llamado Plan de Mejoramiento Educativo (PME). De esta manera se asocian resultados a un modelo de gestión de recursos. Con esto se amarra el funcionamiento y gestión de las escuelas a los resultados alcanzados en este tipo de pruebas. Todos estos aspectos van quitando autonomía a las escuelas y otorgando mayor poder a las pruebas estandarizadas que asumen el control del sistema educativo.

9 Ver más en http://portales.mineduc.cl/usuarios/convivencia escolar/doc/201 103050058380.Anexo\%201\%20Resumen\%20 Ley\%20SEP.pdf

\section{Análisis y discusión: reflexiones del proceso privatizador}

Hemos descrito hasta aquí el centro de una problemática compleja situando a las familias frente a un problema social que tensiona finalidades educativas versus finalidades económicas. Hemos mostrado como la promesa de movilidad social a través de la educación ha sido incumplida, por medio de una política de privatización educativa donde se combinan estrategias de privatización abierta e interna (Bellei, 2015).

Esta proposición supera la definición binaria entre educación pública y privada, propuesta por UNESCO, la que entiende que "escuela pública" se refiere a las instituciones que son operadas por autoridades del Estado, es decir el control es realizado por autoridades públicas; empero, al interior de las instituciones se adquiere un modo de funcionamiento privado, gracias a la entrada de sistemas de administración y gerenciamiento privado. Formas de planificar la enseñanza y las evaluaciones son realizadas por instituciones privadas. En este ejercicio la escuela pública busca asimilarse a la educación privada, creándose una competencia abiertamente desleal. En definitiva, este es un fenómeno que debe ser estudiado con mayor precisión, dado que:

En rigor no se trata de una privatización del sistema de educación pública, sino de una privatización del sistema educacional, por cuanto incrementa la presencia de la educación privada sin que esto implique necesariamente una transformación de las escuelas públicas existentes (Bellei, 2015, p. 65).

Este silencioso proceso es preocupante no solo porque ha modificado las relaciones entre lo que se entiende como "lo público" y "lo privado" -aquello que tiene su sentido común afianzado en la comunidad, diferente de aquello que responderá en última instancia, a intereses particulares -. Esta relación de privatización silenciosa, tensiona al sistema, favoreciendo a las escuelas privadas ya que además de tener el derecho de seleccionar y 
discriminar a sus estudiantes, su modo de funcionar está orientado a un modelo de negocios que buscar generar lucro, quedando las familias en medio de estas operaciones.

Observamos cómo la normativa en evaluación convertida en un cuchillo que agranda la herida apoya este proceso económico, construyendo por medio de sus prácticas de exclusión y segregación, subjetividades vinculadas al mercado y la competencia. De fondo, las prácticas evaluativas ponen el foco en la producción de una subjetividad de mercado, produciendo modelo de matriz colonial, que utiliza a la familia como objeto de gobierno clave en los procesos de estructuración social:

Podemos concluir entonces que "el huérfano" por excelencia de la pedagogía de la dominación no es solo el niño, sino el niño de la periferia, el huérfano colonial, neocolonial, el mestizo latinoamericano al que le introyectan gato (cultura imperial) por liebre (naturaleza humana) (Dussel, 1980, pp. 38-39).

No solo son los estudiantes, en general, más allá de su condición de niñez y juventud, los que sufren esta deformación de horizontes educativos, son también los docentes que particularmente son instrumentalizados para que este modelo de dominación funcione con mayor encubrimiento. Nuestra cultura latinoamericana es oscurecida, negada por este momento ontológico basado en un relato inventado en la Modernidad, la vieja promesa latinoamericana.

El problema de la evaluación va cruzando todos estos temas casi invisiblemente, coloniza todas las esferas de la vida cotidiana, interiorizando un modo de ser individual que penetra la estructura de las familias, moviéndolas a aceptar este modelo individualizante que los responsabiliza de sus resultados académicos. En un principio se hace parte de nuestros cuerpos dóciles, por medio de las múltiples prácticas evaluativas dispuestas en la escuela; más tarde, en el afuera de la sociedad nos controla como dispositivo biopolítico, ya que es por medio de esta técnica de poder gobierna las decisiones que la familia deberá tomar, ejerciendo un control a distancia sobre la vida.

Este control interno, implicado en nuestra estructuración colonial resulta ser inmanente a la forma en que nos reconocemos, a nuestra forma de habitar, conocer y situarnos frente al mundo y dentro de él, es aquello que los autores del enfoque decolonial refieren como "colonialidad del poder" (Restrepo y Rojas, 2010) que opera en cada uno de los espacios, ámbitos, dimensiones materiales y simbólicas de la sociedad.

Hemos destacado un proceso que, por medio de normativas y reglamentos, ha configurado un fenómeno que ha dejado en la orfandad del mercado a las familias, y que pese a ello aún mantienen la esperanza de promoción social. Sin embargo, esta situación no agota el problema, ya que la entrada a la escuela de un niño o niña implica una renuncia a aquello que ha constituido su soporte cultural. Todo el sistema de pensamiento social que portan los estudiantes producto de su socialización primaria entra en conflicto con los códigos culturales que sustenta la escuela, lo que es evidente al observar lo dificultoso que se hace para los estudiantes avanzar en el entretejido cultural del cual está hecha la escuela. Para un estudiante de origen socioeconómico popular, este proceso de renuncia es aún más radical, ya que posee menos herramientas que un estudiante con mayor capital cultural para enfrentar este proceso de asimilación cultural, entonces su mejor manera de adaptarse es sacarse buenas calificaciones, que lo habiliten en el flujo de los méritos individuales y que lo ayude a igualarse entre los iguales.

Vemos este fenómeno no solo desde el prisma económico, sino que lo reconocemos también como un problema cultural, ya que la escuela es impensable fuera de procesos civilizatorios $y$, a la vez, es impensable sin calificaciones, estas últimas son el mecanismo de agenciamiento social y cultural que despoja a las familias de sus formas culturales, alentados por la idea de progreso, que siempre está delante de sus ojos con los brillos de un neón que no deja de encandilar. Es hora ya de pensar, la escuela fuera de estas elecciones de 
mercado, de manera tal que prometa otros horizontes de justicia.

\section{Referencias bibliográficas}

Bellei, C. (2015). El gran experimento. Santiago: LOM. Carlino, F. R. (1999). La evaluación educacional. Historia, problemas y propuestas. Buenos Aires: Aique editorial.

CEPAL (2011). Informe estadístico 2011. (2013). Informe estadístico 2014.

CEPAL-UNESCO (1990). Transformación productiva con equidad. Santiago: UNESCO.

(1992). Educación y conocimiento: eje de la transformación productiva con equidad. Santiago: UNESCO.

De Sousa Santos, B. (2011). Epistemologías del Sur. Utopía y Praxis Latinoamericana. Revista Internacional de Filosofía Iberoamericana $y$ Teoría Social, 16(54), 17-39.

(2013). Descolonizar el saber, reinventar el poder. Santiago: LOM.

Dussel, E. (1980). La pedagógica latinoamericana. Red de Bibliotecas Virtuales de CLACSO. Recuperado de http://biblioteca.clacso.edu.ar

Elliot, J. (2002). The Paradox of Educational Reform in the Evaluatory State: Implications for Teacher Education. Recuperado de http://www.researchgate.net/publication/225971650.

Fernández, D. E. \& Bernasconi, A. (2012). Elementos conceptuales para el análisis organizacional de universidades en contextos de mercado. Innovar, 22(46), 87-98.

Flórez, T. (2011). La importancia de una perspectiva histórica para el análisis de las políticas educativas (o de cómo llevamos haciendo lo mismo durante mucho tiempo). Revista Historia 2.0, Conocimiento histórico en clave digital (2). Recuperado de http://historiaabierta.org/historia2.0/index.php/revista/article/view/31

Foucault, M. (2004). El nacimiento de la biopolítica. México: Fondo de Cultura Económica.

Foucault, M. (2005). Lecciones sobre la voluntad de saber. Buenos Aires: Siglo XXI.

Gysling, J. (2015). The historical development of educational assessment in Chile:
1810-2014. Assessment in Education: Principles, Policy \& Practice, DOI: 10.1080/0969594X.2015.1046812

OCDE (2014a). Income Inequality. Recuperado de http://data.oecd.org/inequality/incomeinequality.htm

(2014b). Panorama Educación Indicadores de la OCDE 2014. Recuperado de http:// www.mecd.gob.es/dctm/inee/indicadoreseducativos/panorama2014/panorama-dela-educacion-2014informe-espanol-05-sep-. pdf?documentId $=0901 e 72 b 81 a 722 a c$

Restrepo, E. \& Rojas, A. (2010). La inflexión decolonial. Fuentes, conceptos y cuestionamientos. Popayán, Colombia: Editorial Universidad del Cauca.

Ruiz, C. (2010). De la República al mercado. Santiago: LOM.

Stobart, G. (2010). Tiempos de pruebas: Los usos y abusos de la evaluación. Madrid: Editorial Morata.

\section{Webgrafía}

http://www.agenciaeducacion.cl/nosotros/quienessomos/

http://www.agenciaeducacion.cl/nosotros/quienessomos/

http://ciperchile.cl/2014/12/01/por-que-se-elige-particular-subvencionado/

http://www.curriculumenlineamineduc.cl/605/ w3-article-33859.html

http://www.leychile.cl/Navegar?idNorma $=30330$

http://www.leychile.cl/Navegar?idNorma $=1006043$

http://www.memoriachilena.cl/602/w3-article-719. html\#cronologia

http://www.fundacionsol.cl/2015/09/la-violenciaestructural-y-cotidiana-a-42-anos-del-golpe-los-11-pilares-dictatoriales-que-todaviasostienen-el-modelo-economico-social/

http://portales.mineduc.cl/usuarios/convivencia_ escolar/doc/201103050058380.Anexo\%20 1\%20Resumen\%20Ley\%20SEP.pdf

http://www.leychile.cl/Navegar?idNorma $=242302$

http://www.leychile.cl/Navegar?idNorma=70774 


\section{Anexos}

\section{Tabla 1. Normativas consideradas en el estudio}

\begin{tabular}{|c|c|c|}
\hline Año & Tipo de Normativa & Principales disposiciones \\
\hline 1860 & $\begin{array}{l}\text { Ley General de } \\
\text { Educación Primaria }\end{array}$ & $\begin{array}{l}\text { Regula el sistema educativo en niveles de educación primaria y segundaria. http://www. } \\
\text { archivonacional.cl/616/w3-article-28319.html }\end{array}$ \\
\hline 1920 & Ley Orgánica 3.654 & $\begin{array}{l}\text { Ley de Instrucción primaria. http://www.memoriachilena.cl/archivos2/pdfs/MC0018131. } \\
\text { pdf }\end{array}$ \\
\hline 1927 & Decreto 6.087 & Estatuto de los exámenes de Educación Secundaria. \\
\hline 1929 & $\begin{array}{l}\text { Decreto de Ley } \\
3.060\end{array}$ & $\begin{array}{l}\text { Disposiciones en favor de regular sistema de evaluación para la educación primaria. } \\
\text { http://www.leychile.cl/Navegar?idNorma=129136 }\end{array}$ \\
\hline 1929 & Decreto Ley 2.545 & Disposición sobre los grados de educación secundaria y exámenes. \\
\hline 1937 & Circular $N^{\circ} 77$ & $\begin{array}{l}\text { Disposición sobre los exámenes y otras actividades finales y sobre la planificación del } \\
\text { próximo año escolar que afecta a la educación primaria. }\end{array}$ \\
\hline 1944 & Decreto Ley 2.944 & Disposición sobre los exámenes y promoción para los alumnos de educación secundaria. \\
\hline 1949 & Decreto Ley 1469 & Disposición sobre los exámenes y promoción para los alumnos de educación secundaria. \\
\hline 1959 & Decreto 842 & $\begin{array}{l}\text { Disposición sobre calificaciones, exámenes y promoción para los alumnos de educación } \\
\text { secundaria. }\end{array}$ \\
\hline 1965 & Decreto 27.952 & Reforma educativa que incluye obligatoriedad escolar por ocho años. \\
\hline 1966 & Decreto 11.207 & $\begin{array}{l}\text { Disposiciones para la evaluación y promoción de séptimo grado para los alumnos de } \\
\text { Educación General Básica. }\end{array}$ \\
\hline 1967 & Decreto 7056 & $\begin{array}{l}\text { Disposiciones para la evaluación y la promoción de alumnos de octavo grado de educa- } \\
\text { ción general y la base para la certificación. }\end{array}$ \\
\hline 1968 & Decreto 6.859 & $\begin{array}{l}\text { Disposiciones para la evaluación y la promoción de octavo grado de educación general } \\
\text { y la base para la certificación. }\end{array}$ \\
\hline 1972 & Decreto 192 & Reglamento para la evaluación y promoción de los alumnos de educación básica. \\
\hline 1974 & $\begin{array}{l}\text { Decreto } \\
\text { Supremo } 164\end{array}$ & $\begin{array}{l}\text { Disposiciones sobre promoción y de la evaluación en sistema educativo. http://www. } \\
\text { leychile.cl/Navegar?idNorma=251386 }\end{array}$ \\
\hline 1975 & Decreto 440 & $\begin{array}{l}\text { Disposiciones sobre promoción y de la evaluación en sistema educativo. http://www. } \\
\text { leychile.cl/Navegar?idNorma=251386 }\end{array}$ \\
\hline 1978 & Decreto 2.038 & $\begin{array}{l}\text { Disposiciones sobre promoción y de la evaluación en sistema educativo. http://www. } \\
\text { leychile.cl/Navegar?idNorma=251386 }\end{array}$ \\
\hline 1979 & Decreto 2.088 & $\begin{array}{l}\text { Disposiciones sobre promoción y de la evaluación en sistema educativo. http://www. } \\
\text { leychile.cl/Navegar?idNorma=251386 }\end{array}$ \\
\hline 1990 & $\begin{array}{l}\text { Ley Orgánica Cons- } \\
\text { titucional } 18.962\end{array}$ & $\begin{array}{l}\text { Ley Orgánica Constitucional de Enseñanza, permite publicación de resultados de prue- } \\
\text { bas nacionales (SIMCE). https://www.leychile.cl/Navegar?idNorma=247551 }\end{array}$ \\
\hline 2011 & Ley 20.529 & $\begin{array}{l}\text { Sistema Nacional de Aseguramiento de la Calidad de la Educación, permite fiscaliza- } \\
\text { ción de las escuelas e intervención según resultados SIMCE. http://www.leychile.cl/ } \\
\text { Navegar?idNorma }=1028635\end{array}$ \\
\hline 2008 & Ley 20.248 & $\begin{array}{l}\text { Ley Subvención Escolar Preferencial, asigna subsidios económicos por resultados obte- } \\
\text { nidos en pruebas nacionales. https://www.leychile.cl/Navegar?idNorma=269001 }\end{array}$ \\
\hline
\end{tabular}

